\title{
TLR7 Contributes to the Rapid Progression but Not to the Overall Fatal Outcome of Secondary Pneumococcal Disease following Influenza A Virus Infection
}

\author{
Sabine Stegemann-Koniszewski ${ }^{a}$ d Marcus Gereke ${ }^{a, e}$ Sofia Orrskog ${ }^{g}$ Stefan Lienenklaus ${ }^{b}$ \\ Bastian Pasche ${ }^{c}$ Sophie R. Bader ${ }^{f}$ Achim D. Gruber $^{f}$ Shizuo Akira ${ }^{h}$ Siegfried Weiss ${ }^{b}$ \\ Birgitta Henriques-Normark ${ }^{g}$ Dunja Bruder ${ }^{a}$ e Matthias Gunzer $^{d}$ \\ ${ }^{a}$ Immune Regulation Group, ${ }^{b}$ Molecular Immunology and CInfection Genetics, Helmholtz Centre for Infection Research, \\ Braunschweig, ${ }^{d}$ Institute of Experimental Immunology and Imaging, University Clinic, University of Duisburg-Essen, Essen, \\ e Infection Immunology Group, Department of Medical Microbiology, Otto von Guericke University Magdeburg, Magdeburg, and \\ ${ }^{f}$ Department of Veterinary Pathology, Freie Universität Berlin, Berlin, Germany; ${ }^{9}$ Department of Microbiology, Tumor and Cell \\ Biology, Karolinska Institute, Stockholm, Sweden; ${ }^{h}$ Department of Host Defence, Research Institute for Microbial Diseases, Osaka \\ University, Osaka, Japan
}

\section{Key Words}

Toll-like receptor 7 - Innate immunity - Influenza A virus • Streptococcus pneumoniae $\cdot$ Bacterial superinfection

\begin{abstract}
Increased risk for bacterial superinfections substantially contributes to the mortality caused by influenza $A$ virus (IAV) epidemics. While the mechanistic basis for this lethal synergism is still insufficiently understood, immune modulation through the viral infection has been shown to be involved. Since the pattern-recognition receptor (PRR) toll-like receptor 7 (TLR7) is a major sensor for the viral genome, we studied how IAV recognition by TLR7 influences the development of secondary pneumococcal infection. In a mouse model of IAV, TLR7-deficient hosts induced a potent antiviral response and showed unchanged survival. In secondary pneumococcal infection during acute influenza, TLR7ko mice showed a fatal outcome similar to wild-type (WT) hosts, despite significantly delayed disease progression. Also, when bacterial superinfection occurred after virus clearance, WT and TLR7deficient hosts showed similar mortality, even though we found the phagocytic activity of alveolar macrophages iso-
\end{abstract}

lated from IAV-pre-infected hosts to be enhanced in TLR7ko over WT mice. Thus, we show that a virus-sensing PRR modulates the progression of secondary pneumococcal infection following IAV. However, the fatal overall outcome in WT as well as TLR7ko hosts suggests that processes distinct from TLR7-triggering override the contribution of this single PRR.

Copyright $\odot 2012$ S. Karger AG, Basel

\section{Introduction}

A retrospective study of the 1918/19 Spanish flu has shown that the majority of fatalities were not due to the primary infection with influenza A virus (IAV) but rather severe bacterial superinfections. In these, Streptococcus pneumoniae was the most common bacterial pathogen [1]. This also holds true for later pandemics, including the swine flu outbreak in 2009 [2-4]. A conclusive molecular explanation of how a previous IAV infection can leave the host's antibacterial defense in a paralyzed

D. Bruder and M. Gunzer contributed equally to the study.

\section{KARGER}

Fax +4161306 1234

E-Mail karger@karger.ch

www.karger.com (c) 2012 S. Karger AG, Basel

1662-811X/13/0051-0084\$38.00/0

Accessible online at:

www.karger.com/jin
Prof. Dr. Matthias Gunzer

Experimental Immunology and Imaging, Centre for Medical Biotechnology Universitätsstrasse 2

DE-45117 Essen (Germany)

E-Mail matthias.gunzer@uni-due.de 
state for up to several months has not yet been found [5]. However, several mechanisms by which IAV infection modulates the immune system, rendering it incapable of mounting an adequate antibacterial response, have been revealed lately [6-9]. Yet, by which molecular recognition events this immune modulation is triggered has remained elusive. Clearly, a detailed understanding of the underlying mechanisms will be required to meet the threat of new influenza pandemics in combination with the increasing number of circulating antibiotic-resistant strains of relevant bacterial pathogens.

The innate immune system possesses pattern recognition receptors (PRR) that bind conserved structures of pathogenic microorganisms and trigger defined immune reactions. An extensively studied group of these receptors is the toll-like receptor (TLR) family comprising TLRs $1-11$, which are critically linked to the defense against various pathogens $[10,11]$. The single-stranded negativesense IAV genome is recognized by TLR7 and 8, which are localized in the endosomal membrane of expressing cells $[12,13]$. Triggering of TLR7 leads to the release of cytokines and type I IFNs, and can mediate profound local and systemic effects, e.g. the induction of peripheral blood lymphopenia and modulation of immune responses $[14,15]$. Previous studies in IAV-infected animals have revealed that in the absence of the adaptor protein MyD88, signaling through RIG-I is sufficient for mounting effective initial immune responses and that selective TLR7deficiency has distinct effects on shaping adaptive immunity [16-20]. Regarding the contribution of TLR7 to the innate responses following IAV infection, increased accumulation of myeloid-derived suppressor cells in the lungs of TLR7ko animals has recently been shown [18]. However, the potential role of TLR7 in bacterial superinfection following influenza has not been addressed so far. While these are most commonly recognized during ongoing IAV infections, other respiratory viruses also predispose for secondary bacterial complications. These include rhinoviruses, respiratory syncytial virus and measles virus [21,22]. Interestingly, most of these viruses possess a single-stranded RNA genome and therefore potentially supply a TLR7-trigger $[23,24]$. This makes it conceivable that TLR7 triggering during the viral infection mediates some of the immune-modulatory effects which subsequently facilitate bacterial superinfections.

Therefore, in order to clarify how TLR7-mediated antiIAV responses possibly impact the synergism between respiratory viral and secondary bacterial infections, we have analyzed TLR7-deficient mice during experimental IAV infection and co-infection with S. pneumoniae. As others, we find that the course of IAV infection is not strikingly altered in TLR7 deficient hosts. Even though highly conceivable, we did not find TLR7 to modulate the fatal outcome of bacterial superinfection during influenza. Nevertheless, the course of pneumococcal disease occurring during acute IAV infection was significantly delayed in TLR7-deficient hosts. Therefore, our work shows that TLR7 as a virus-sensing PRR, even though not a major contributor, constitutes one of the many factors involved in the synergism of IAV and S. pneumoniae in mammals.

\section{Materials and Methods}

Mice

TLR7ko (TLR7 ${ }^{-/-}$) mice [25], back-crossed at least five generations to the C57BL/6 background, were a gift from S. Bauer (Marburg, Germany) and were bred at the animal facility of the Helmholtz Centre for Infection Research (HZI). Age- and sex-matched littermates were used as WT controls in experiments with five generation back-crossed TLR7ko mice. C57BL/6 mice were purchased from Charles River (Sulzfeld, Germany) or Harlan Winkelmann (Borchen, Germany) and used as controls in experiments with ten generation back-crossed TLR7ko. For luciferase reporter mice, IFN- $\beta^{\Delta \beta \text {-luc/ } \Delta \beta \text {-luc }}$ males were crossed with TLR7 $^{-/-}$females to obtain TLR7 ${ }^{-/ y}$ IFN- $\beta^{+/ \Delta \beta-l u c}$ males or C57BL/6 females to obtain TLR7 $7^{+/ y}$ IFN- $\beta^{+/ \Delta \beta-l u c}$ control males.

All animal experiments were approved by the Niedersächsisches Landesamt für Verbraucherschutz und Lebensmittelsicherheit (file No. 33.42502-006/07) for the HZI and the Swedish animal welfare authority (file No. N254/07) for the Karolinska Institute, and were conducted in conformity with the German animal welfare act, the Swedish animal protection legislation and the European Communities Council Directives 86/609/EEC and 2010/63/EU.

\section{Viral and Bacterial Pathogens}

Madin-Darby canine kidney cell-derived IAV PR8/A/34 (H1N1) was obtained as described [26]. S. pneumoniae TIGR4 was grown on blood agar (Columbia agar with $5 \%$ sheep blood; $\mathrm{BD}$, San Jose, Calif., USA) at $37^{\circ} \mathrm{C}$ and $5 \% \mathrm{CO}_{2}$. Colonies were inoculated into $\mathrm{C}+\mathrm{Y}$ (casamino acid and yeast extract) or THY (Todd Hewitt Broth, $1 \%$ yeast extract) medium, grown to midlogarithmic phase and diluted to the appropriate concentrations. For S. pneumoniae, bacterial counts in the inoculum used for mouse infection were confirmed by plating serial dilutions.

\section{Viral and Bacterial Infections}

For mouse infections, 8- to 12-week-old mice were anaesthetized by isoflurane inhalation or intraperitoneal injection of ketamine/ xylozine in the case of luciferase reporter mice. A volume of $25 \mu \mathrm{l}$ (IAV) or $20 \mu \mathrm{l}$ (S. pneumoniae) containing the appropriate concentration of virus or bacteria was administered onto the nostrils. For IAV, the dose lethal to $50 \%$ of inoculated C57BL/6 mice (MLD50) was determined as previously reported [26]. For S. pneumoniae, bacteria were diluted in growth medium to $5 \times 10^{6} \mathrm{CFU} / \mathrm{ml}$. During all mouse infection experiments, animals were sacrificed when observing overt signs of illness or weight loss of more than $25 \%$. 


\section{Lung Viral Load}

Lungs were homogenized in TriFastFL reagent (PeqLab). RNA was extracted by addition of 1-Br-3-Cl-Propane (Merck), purified by precipitation and treated with DNase (Ambion) prior to reverse transcription to cDNA (M-MLV Reverse Transcriptase; Invitrogen, Carlsbad, Calif., USA). Quantitative real-time PCR of $100 \mathrm{ng}$ cDNA samples was conducted using the Brilliant SYBR Green QPCR Kit (Stratagene) and primers specific for the IAV nucleoprotein (NP). Quantitative real-time PCR of samples containing known numbers of a plasmid carrying the NP sequence (pVIPmH5-PR8-NP; provided by G. Sutter, Munich, Germany) was performed to obtain a CT-value/NP copy number standard, which was used to quantify NP copy numbers in the samples.

Quantification of IFN- $\gamma$ in Bronchoalveolar Lavage

Lungs were flushed with $1 \mathrm{ml}$ PBS and a Luminex-based immunoassay (Millipore) was used according to the manufacturer's recommendations. Samples were acquired on a LiquiChip 100 Workstation (Qiagen) and data were analyzed using the LiquiChip Analyzer 1.0.

Quantification of Leukocytes in Bronchoalveolar Lavage

Lungs were flushed with $1 \mathrm{ml}$ PBS and cells from bronchoalveolar lavage (BAL) were collected by centrifugation. Erythrocytes were lysed by osmotic shock and viable cells were counted to assess the total number of cells/ml BAL. Fc- $\gamma$ receptor block was performed through incubation with anti-mouse CD16/CD36 (2.4G2) antibody. Cells were stained for CD11b (M1/70), Gr-1 (RB6-8C5), F4/80 (BM8), CD4 (RM4-5) and CD8 (53-6.7) (BD/eBioscience). For analysis, cells were analyzed for $\mathrm{Gr} 1^{\text {high }} / \mathrm{CD} 11 \mathrm{~b}^{+}$neutrophils following the exclusion of macrophages (F4/80 $0^{+}$cells). For each population, the absolute cell number was calculated from the percent population data from flow cytometric analysis.

\section{Flow Cytometry}

Flow-cytometric analyses were performed using a FACS Canto instrument (BD) and FACS Diva (BD), Summit (DAKO) or FlowJo (Tree Star) software.

\section{Assessment of S. Pneumoniae CFU Counts}

Blood $(5 \mu \mathrm{l})$ from the tail vein was diluted in PBS and plated on blood agar plates. Lungs were collected in $1 \mathrm{ml}$ of PBS, homogenized and serial dilutions were plated. $\mathrm{CFU}$ were counted after $16 \mathrm{~h}$ of incubation at $37^{\circ} \mathrm{C}$ and $5 \% \mathrm{CO}_{2}$.

\section{Histology}

Lungs were fixed in $4 \%$ paraformaldehyde, followed by paraffin embedding, preparation of $4-\mu \mathrm{m}$ sections and staining with hematoxylin-eosin. Histological evaluation was performed by an animal pathologist certified by the European College of Veterinary Pathologists in a blinded fashion.

Detection of Luciferase Activity in IFN- $\beta^{+/ \Delta \beta-l u c}$ Mice

Mice were injected with R-848 in PBS (50 $\mu$ g i.v.; Invivogen, San Diego, Calif., USA) or intranasally infected with IAV. Detection of luciferase activity was performed by in vivo imaging or ex vivo quantification in lung homogenates as reported previously [27].

In vivo Phagocytosis Assay

S. pneumoniae TIGR4 was grown on blood agar plates. Bacteria were resuspended in PBS, pelleted by centrifugation and incu- bated in $0.1 \mathrm{mg} / \mathrm{ml}$ fluorescein isothiocyanate (FITC) in $0.1 \mathrm{M}$ $\mathrm{NaHCO}_{3}(\mathrm{pH}$ 9) for $1 \mathrm{~h}$. Bacteria were washed and resuspended in PBS to $\sim 1.25 \times 10^{8} \mathrm{CFU} / \mathrm{ml}$ and used for intranasal inoculation of anaesthetized mice $(20 \mu \mathrm{l})$. Animals were sacrificed $2 \mathrm{~h}$ later and lungs were flushed twice with a total volume of $2 \mathrm{ml}$ PBS. Cells from BAL were collected by centrifugation, erythrocyte lysis and Fc- $\gamma$ receptor block were performed and cells were stained for F4/80 followed by flow-cytometric analysis.

\section{Statistical Analysis}

Statistics were performed by the indicated t tests and survival data were compared by Kaplan Meier analysis log-rank test using Graph Pad Prism 5.00 (Graph Pad Software, La Jolla, Calif., USA).

\section{Results}

\section{Increased Body-Weight Loss but Otherwise Unaltered} Course of IAV Infection under TLR7 Deficiency

In order to address the response to secondary bacterial infection after sublethal IAV infection in the absence of TLR7, we first characterized survival and weight loss of TLR7ko mice after the viral infection alone. We chose the viral strain A/PR8/34 (H1N1) as an extensively studied IAV representative. Following IAV infection of wildtype (WT) and TLR7ko mice with $0.04 \mathrm{LD}_{50}$ PR8 virus, no significant differences in survival were detectable (fig. 1a). To address a possible effect of TLR7 present only during infection with higher viral doses, survival following infection with $1.0 \mathrm{LD}_{50}$ PR8 virus was assessed in both mouse strains. Also here, no effect of TLR7-deficiency became apparent (fig. 1a).

When comparing the body weight loss in the course of IAV infection between both mouse strains, a subtle TLR7dependent exacerbation was apparent (fig. 1b). We observed increased early weight loss and delayed recovery to the original body weight in TLR7ko animals at both the viral doses tested, which confirms results recently published by Jeisy-Scott et al. [18].

To assess viral clearance in TLR7ko animals, viral NP RNA sequence copy numbers were quantified in the course of infection with $0.04 \mathrm{LD}_{50}$ IAV (fig. 1c). For these and the following experiments characterizing the antiIAV response in TLR7ko mice, the lower viral dose was chosen to ensure survival and recovery of nearly all animals within experimental groups. Independent of the presence or absence of TLR7, IAV was cleared with similar kinetics in the lungs of both mouse strains. Taken together, these analyses confirmed previous studies $[18,19]$ that showed that TLR7 is dispensable for survival and viral clearance following respiratory IAV infection. 


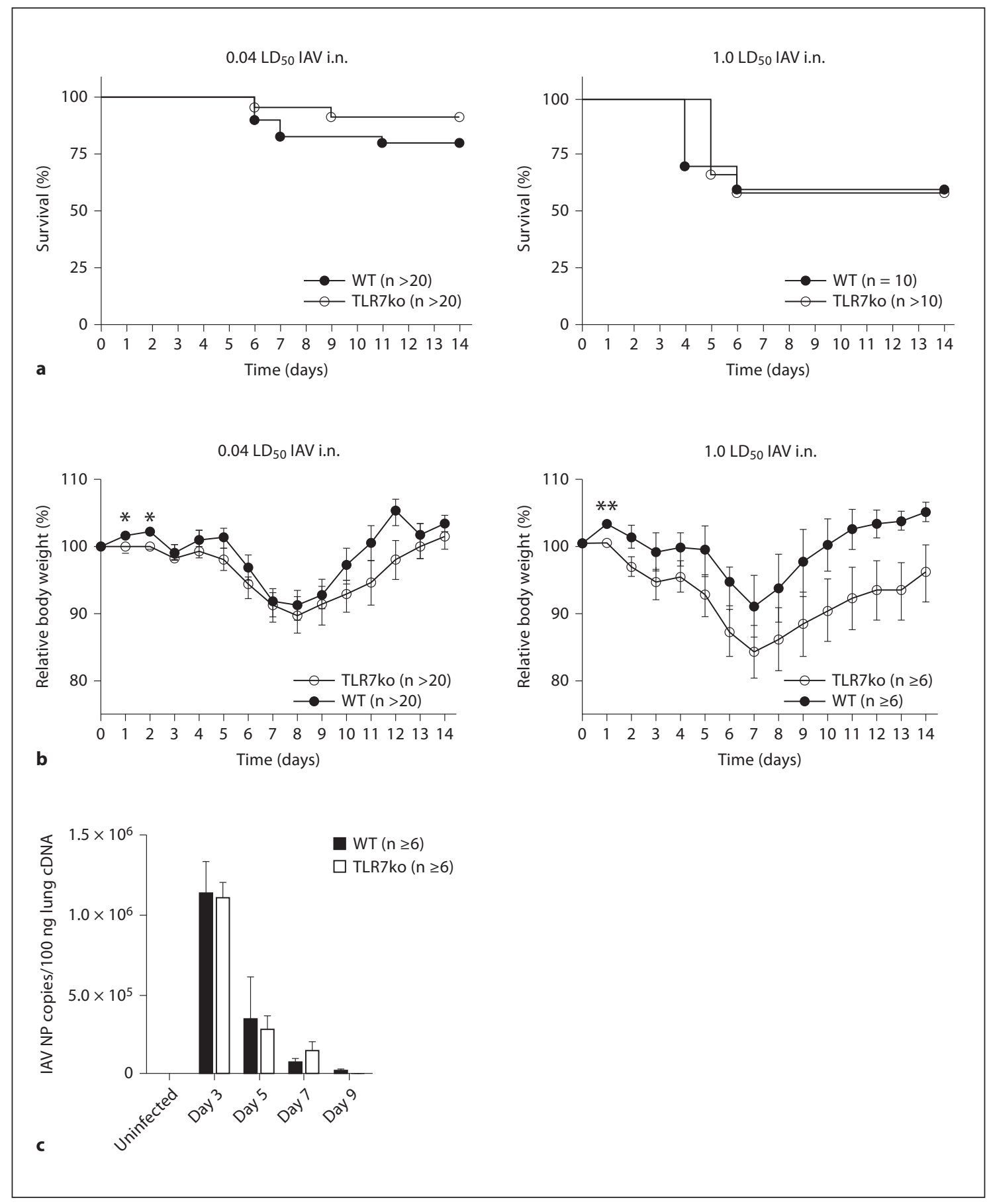

Fig. 1. TLR7 deficiency leaves survival and viral clearance following IAV infection unaffected but exacerbates body weight loss. a WT and TLR7ko mice were intranasally infected with $0.04 \mathrm{LD}_{50}$ or $1.0 \mathrm{LD}_{50}$ influenza PR8 and body weight and survival were assessed over 14 days. $\mathbf{b}$ The relative body weight is shown as mean \pm SEM (no error bars shown for SEM $<1 \%$ ) of all animals surviving the infection to day $14\left[{ }^{*} \mathrm{p}=0.027\left(0.04 \mathrm{LD}_{50}\right.\right.$ day 1$)$ and 0.007
(0.04 $\mathrm{LD}_{50}$ day 2$),{ }^{* *} \mathrm{p}<0.001\left(1.0 \mathrm{LD}_{50}\right.$ day 1$)$ as determined by an unpaired, two-sided t test]. c For quantification of airway viral load in WT and TLR7ko mice, lung cDNA was analyzed for influenza NP sequence copy numbers following infection of WT and TLR7ko mice with $0.04 \mathrm{LD}_{50}$ IAV PR8. Data are shown as mean copy numbers \pm SEM ( $n \geq 6$ /group). 


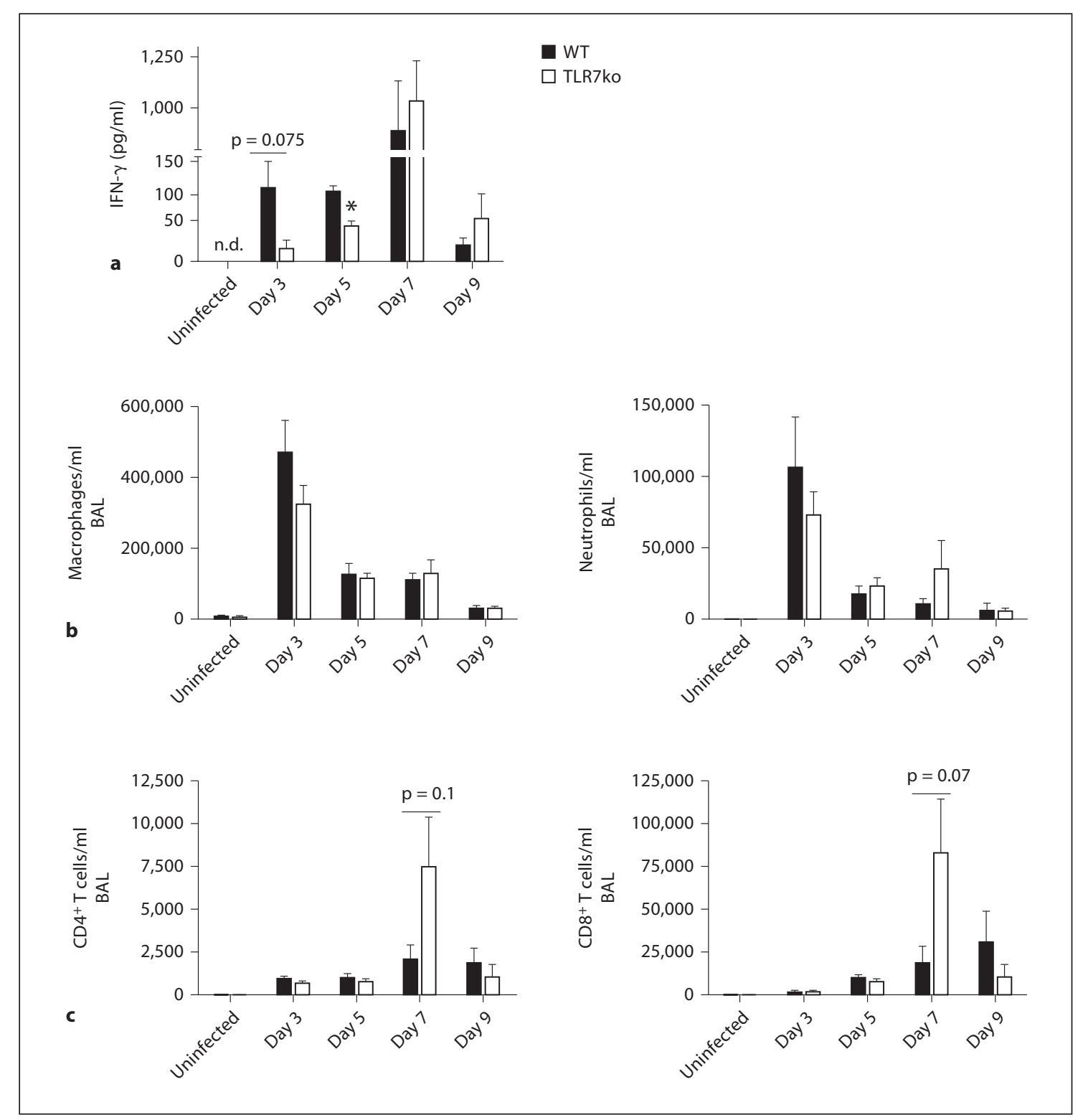

Fig. 2. TLR7 contributes to the early airway IFN- $\gamma$ response but is dispensable for the recruitment of macrophages, neutrophils and $\mathrm{T}$ cells following IAV infection. WT (black bars) and TLR7ko (open bars) mice were infected with 0.04 LD $_{50}$ IAV PR8 and sacrificed at different time points post-infection. a IFN- $\gamma$ concentrations in BAL were determined and protein levels are shown as means \pm SEM of $n \geq 5$ mice/group. The number of cells in BAL was counted and relative contributions of leukocyte subsets assessed by flow-cytometry. Absolute numbers of macrophages, neutrophils (b) as well as $\mathrm{CD}^{+}$and $\mathrm{CD}^{+} \mathrm{T}$ cells (c) were calculated from flow-cytometric data and total cell numbers. Data show mean cell counts/ml \pm SEM of $n \geq 6$ mice/ group and were compared by an unpaired, two-sided t test $\left({ }^{*} \mathrm{p}<\right.$ $0.005)$.

This process is known not to be drastically impaired in TLR7ko hosts $[18,19]$. Of the inflammatory mediators typically released during IAV infection, IFN- $\gamma$ was of special interest to our study of the role of TLR7 in secondary bacterial infection. On the one hand, TLR7ko mice have been found to produce less IFN- $\gamma$ following IAV in- 
fection [18] and on the other hand IFN- $\gamma$ has been shown to be a main contributor in mediating enhanced susceptibility to pneumococci following influenza [7]. Also, in our model of IAV infection, TLR7ko mice revealed impaired IFN- $\gamma$ production in the airways (fig. $2 \mathrm{a}$ ). In serum samples obtained throughout the course of the infection, no significant increase in systemic IFN- $\gamma$ levels could be detected at any of the analyzed time points (data not shown). However, in contrast to previous studies, the inhibition of the airway IFN- $\gamma$ response was evident only at the early time points in the course of infection, but could still possibly have an impact on IAV-induced macrophage inhibition.

As the levels of major chemo-attractants released following IAV infection were not altered by TLR7-deficiency (data not shown) [18], it was an expected finding that also the numbers of macrophages and neutrophils (fig. 2b) in the bronchoalveolar lavage of WT and TLR7 hosts were not significantly altered during the course of infection. Since T cells recruited to the lungs in the course of IAV infection are the major source of airway IFN- $\gamma$ [7], T cell numbers present in bronchoalveolar lavage were assessed over the course of the infection in both WT and TLR7ko mice. $\mathrm{CD}^{+}$and $\mathrm{CD} 8^{+} \mathrm{T}$ cell numbers present in BAL increased over time and there were no significant changes in TLR7ko mice compared to WT (fig. 2c). In addition, $T$ cells isolated from lungs of both mouse strains after infection with PR8 virus showed no difference in in vitro IFN- $\gamma$ production in response to unspecific stimulation (data not shown). This suggests that cellular sources other than $\mathrm{T}$ cells are responsible for the delayed airway IFN- $\gamma$ response in TLR7-deficient hosts. Taken together, we found TLR7 to significantly contribute to the early IFN- $\gamma$ production in the respiratory tract but to be dispensable for potent airway anti-influenza responses such as immune cell recruitment.

\section{TLR7 Deficiency Delays Progression of Pneumococcal} Superinfection following IAV Infection

While the overall outcome of the viral infection was similar in the absence of TLR7, the early lack of IFN- $\gamma$ could possibly influence the anti-bacterial defense in situations of bacterial superinfection, as this cytokine has been found to mediate the functional suppression of alveolar macrophages (AM) [7], which are key cells for anti-bacterial defense. To test this assumption, WT and TLR7-deficient mice were superinfected with S. pneumoniae TIGR4 on day 7 following IAV infection, according to a previously established co-infection model [26]. In this model, mice are infected with a low dose of IAV
PR8 prior to infection with a low dose of S. pneumoniae. We used $0.04 \mathrm{LD}_{50}$ of the virus to be able to clearly distinguish between death through the viral infection, which hardly occurs at this dose (fig. 1a), and death through the secondary bacterial infection. In human patients the primary viral and secondary bacterial infection are also reported to be distinguishable. Typically, patients have nearly recovered from the influenza infection when infection with the secondary bacterial pathogen causes a recurrence of symptoms [31]. When infected with S. pneumoniae 7 days after IAV pre-infection, both WT and TLR7ko mice showed profoundly increased mortality compared to bacterial infection alone without a significant difference between the two mouse strains (fig. 3a). Importantly, survival following S. pneumoniae single infection did not significantly differ between WT and TLR7ko mice (fig. 3a). In the chosen model of pneumococcal infection, death due to bacterial infection is accompanied by the systemic spread of bacteria from the lungs via the bloodstream [32]. Therefore, we assessed the mean time point of the onset of bacteremia after bacterial co-infection as a measure of disease progression. Interestingly, in TLR7ko mice, bacteremia following bacterial superinfection developed at a significantly later point in time when compared to WT mice (fig. 3b). This implied that systemic pneumococcal spread was more efficiently controlled in the absence of TLR7. To exclude that this effect was independent of the viral infection, survival and onset of bacteremia were compared between WT and TLR7ko mice following infection with a higher dose of $S$. pneumoniae alone (online suppl. fig. 1; for all online suppl. material, see www. karger.com/doi/10.1159/000345112). This better resembles the situation in co-infected mice, where high numbers of bacteria are present in the respiratory tract and disseminate through the bloodstream. However, also in the $S$. pneumoniae single infection with a higher dose, no significant difference in survival (online suppl. fig. 1a) or in the onset of bacteremia (online suppl. fig. 1b) was detected between the two mouse strains, suggesting that the results in co-infected mice can be attributed to the underlying IAV infection. In addition to the survival studies in co-infected animals, the lungs and blood of infected WT and TLR7ko mice were harvested at 4 and $24 \mathrm{~h}$ following the bacterial inoculation in order to quantify bacterial CFU. Again, no difference became apparent when comparing WT and TLR7-deficient hosts in the bacterial infection alone (fig. 3c, d). As previously reported and in contrast to naïve animals, IAV-pre-infected WT mice were unable to control bacterial growth 


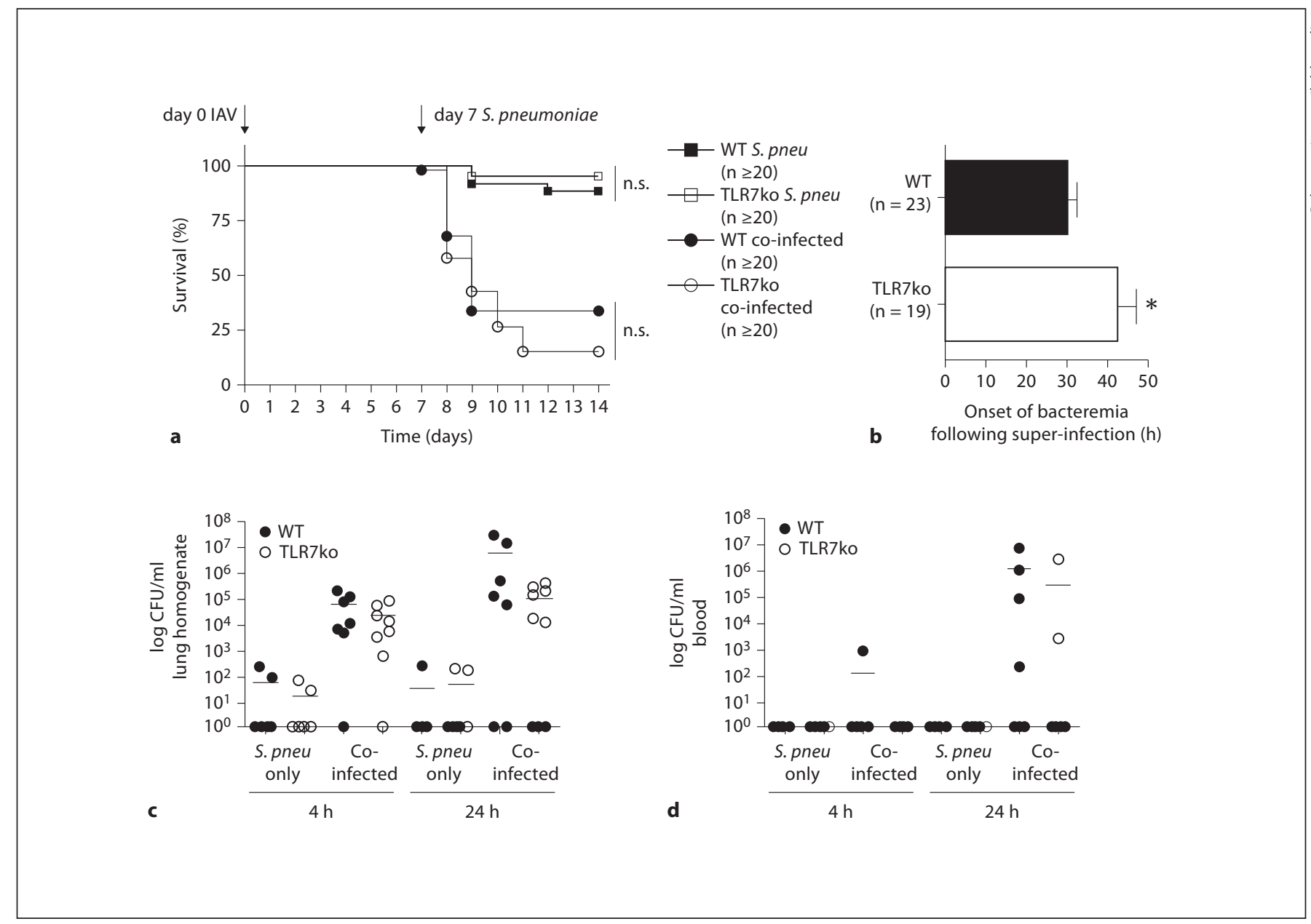

Fig. 3. While survival is not affected, progression of secondary bacterial disease following IAV infection is delayed in TLR7-deficient hosts. WT (closed symbols) and TLR7ko (open symbols) mice were intranasally infected with $0.04 \mathrm{LD}_{50}$ IAV PR8 or left uninfected on day 0 (squares) and infected with $1 \times 10^{5}$ CFU $S$. pneumoniae on day 7 (circles). a Animals were followed for survival over 7 days after the pneumococcal infection and survival was compared by a log-rank test. b Blood samples were collected at 24, 48 and $72 \mathrm{~h}$ and analyzed for the presence of $S$. pneumoniae CFU. The mean time point for onset of bacteremia ( \pm SEM) was calculated from all positively tested mice and compared by unpaired, two-tailed t tests $(\mathrm{p}=0.015)$. In separate experiments, WT (closed circles) and TLR7ko (open circles) mice were sacrificed 4 or $24 \mathrm{~h}$ following the bacterial infection and S. pneumoniae CFU counts in lung homogenates (c) and blood (d) were quantified and compared between mouse strains by unpaired, two-sided t tests. e Histological examination of lungs was performed on uninfected mice at day 7 following IAV infection and $24 \mathrm{~h}$ following co-infection. Blood vessels (V), bronchioles $\left(^{*}\right)$ and intra-alveolar fibrin exudations (arrows) are indicated. Scale bar $=100 \mu \mathrm{m}$. Sections are representative for 2-3 analyzed infected mice per group.

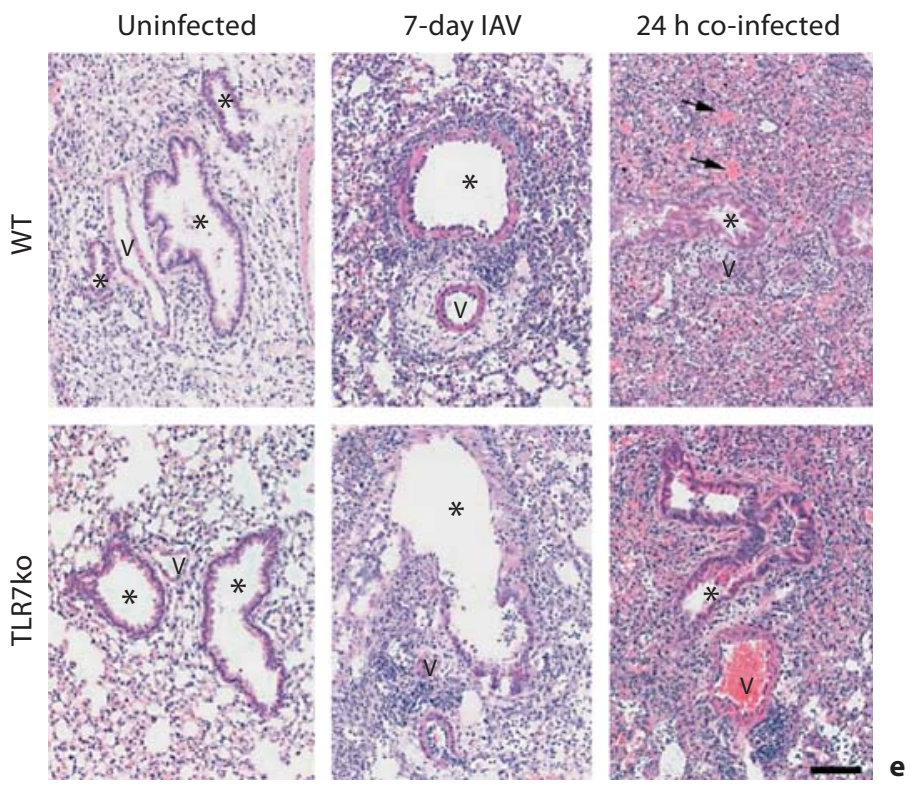




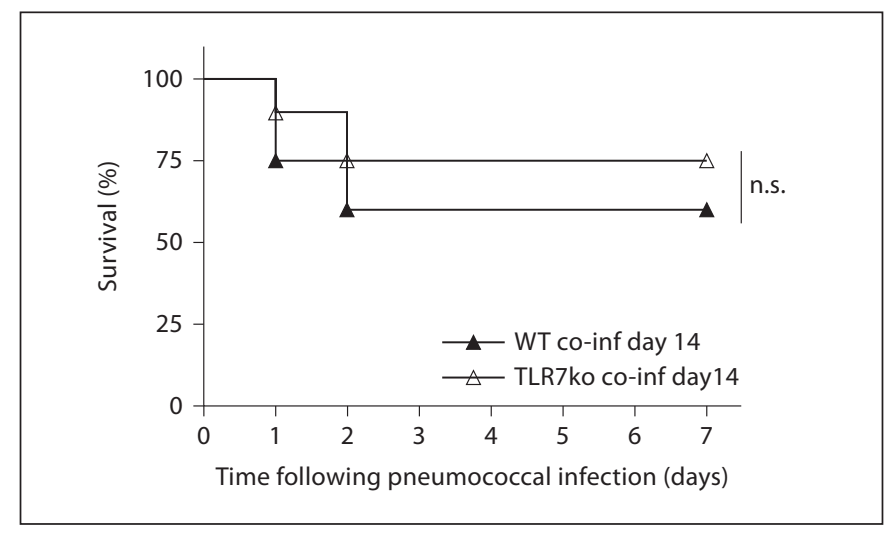

Fig. 4. TLR7 does not influence the outcome of pneumococcal superinfection after recovery from IAV infection. WT $(\mathrm{n}=20)$ and TLR7ko $(\mathrm{n}=20)$ mice were intranasally infected with 0.04 $\mathrm{LD}_{50}$ IAV PR8 14 days before intranasal infection with $1 \times 10^{5}$ CFU S. pneumoniae in at least two independent experiments. Survival was followed over 7 days and compared between mouse strains by a log-rank test. n.s. = Not significant.

in the lower respiratory tract as well as dissemination through the bloodstream [26]. In TLR7ko mice the overall situation was similar. However, co-infected TLR7ko mice, in comparison with co-infected WT mice, carried 55 -fold lower mean bacterial counts in lung tissue $24 \mathrm{~h}$ following pneumococcal infection (fig. 3c). Even though this difference did not reach the level of statistical significance, it was in line with our finding that TLR7 deficiency delayed disease progression and systemic bacterial spread following pneumococcal infection 7 days after IAV infection. This was further supported by the data from blood cultures (fig. 3d) where at 4 and $24 \mathrm{~h}$ following pneumococcal co-infection a higher proportion of WT mice [1/7 (14\%) and 4/7 (57\%), respectively] carried bacteria in their blood when compared to TLR7ko animals [0/9 (0\%) and 2/9 (22\%), respectively]. Histopathology of the lungs of co-infected WT and TLR7ko mice revealed a multifocal to confluent chronic bronchointerstitial pneumonia and perivascular and peribronchiolar lymphocytic cuffings - both consistent with the preceding IAV infection - as well as an accompanying suppurative bronchopneumonia indicating the secondary bacterial infection. There were no significant differences between the lungs of WT and TLR7ko mice concerning the quantity of the lesions. However, in coinfected WT mice the quality of the lung lesions was of a more necrotizing character and showed a more conspicuous intra-alveolar fibrin exudation compared to co-infected TLR7ko mice (fig. 3e).

TLR7 and Pneumococcal Superinfection following Influenza
Taken together, these data show that TLR7 triggering during IAV infection contributes to the rapid progression of bacterial disease occurring during acute influenza. However, TLR7 deficiency was clearly not able to rescue mice from the dramatically high post-IAV susceptibility to an otherwise sublethal dose of $S$. pneumoniae. This highlights the complexity of the exceptional synergism between IAV and bacterial pathogens and the strong impact of additional factors on the underlying mechanisms.

\section{TLR7 Does Not Influence the Outcome of}

Pneumococcal Superinfection after Recovery from IAV Infection

IAV can induce long-term suppression of antibacterial defense mechanisms, rendering hosts highly susceptible to bacterial superinfection for months after clearance of the primary viral infection $[5,8]$. Therefore, we also addressed the susceptibility to $S$. pneumoniae on day 14 following IAV infection. At this time, animals had largely recovered from weight loss and successfully cleared the viral burden. Also here, TLR7ko mice displayed similar survival rates in direct comparison with WT hosts following co-infection on day 14 after IAV (fig. 4).

\section{Altered Type I IFN Levels Are Not Responsible for \\ Delayed Progression of Secondary Bacterial Infection \\ in TLR7ko Hosts}

IAV infection typically induces a strong release of type IIFNs in the infected mammalian host, triggered through the recognition of viral nucleic acids and replication intermediates by PRRs such as TLR7 and RIG-I $[29,33]$. The induced type I IFN response has been shown to be a major contributor to the enhanced susceptibility to postinfluenza bacterial infections [6]. Even though TLR7 deficient hosts are generally capable of mounting an antiviral type I IFN response through alternative innate receptors [19], we wanted to address whether altered levels in the airways of TLR7ko mice in the course of IAV infection possibly accounted for the delay in progression of secondary pneumococcal infection. To overcome the drawbacks of other detection systems, such as insensitivity and the need for invasive sample collection [27], and for an improved understanding of the localization and kinetics of this reaction, we aimed at investigating the type I IFN response after respiratory IAV infection in whole animals. To this end, we took advantage of a luciferase-based IFN- $\beta$ reporter mouse strain [27], which we crossed with TLR7ko mice. The induction of an IFN- $\beta$ response was analyzed through quantification of luciferase activity in vivo or ex vivo. The functionality of the

J Innate Immun 2013;5:84-96 


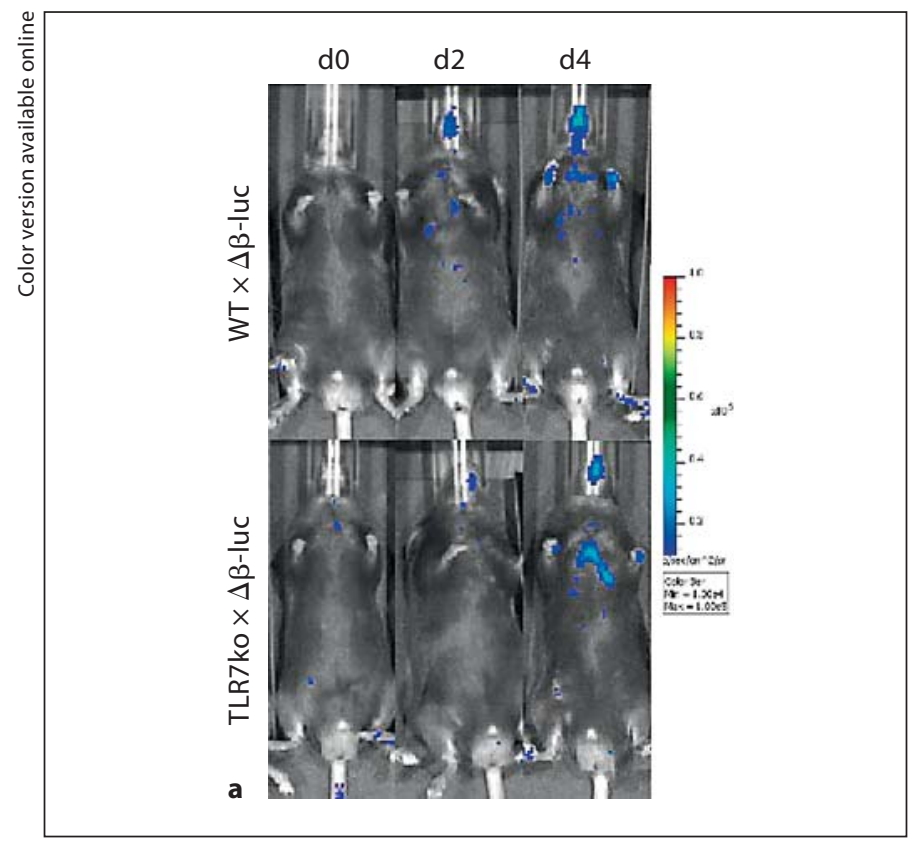

Fig. 5. a Absence of TLR7 does not influence the lung IFN- $\beta$ response following IAV infection. WT and TLR7ko IFN- $\beta^{+/ \Delta \beta-l u c}$ reporter mice were intranasally infected with $0.04 \mathrm{LD}_{50}$ IAV PR8 and induction of luciferase activity was assessed in vivo. One representative of $n \geq 3$ tested animals per mouse strain is shown. b WT (closed circles) and TLR7ko (open circles) IFN- $\beta^{+/ \Delta \beta-l u c}$

reporter strains was confirmed by injection of the TLR7specific ligand R-848, which led to well-detectable luciferase activity exclusively in WT IFN- $\beta^{+/ \Delta \beta \text {-luc }}$ reporter mice (online suppl. fig. S1). However, the in vivo analysis of luciferase activity in IAV infected WT and TLR7ko reporter mice yielded only weak signals, which were hardly sufficient for comparing the two mouse strains (fig. 5a). Thus, to allow accurate quantification of the reporter, luciferase activity was analyzed ex vivo. To this end, lungs were harvested 4 and 7 days after IAV infection. The analysis of lung homogenates confirmed that TLR7ko hosts were able to react to IAV infection by inducing IFN$\beta$ locally in the lung (fig. $5 b$ ). This response was evident at the same time points and reached similar extents as that of WT hosts. Therefore, an altered local type I IFN response by TLR7ko mice in reaction to IAV infection most likely does not account for the alterations found in the progression of secondary pneumococcal infection.

\section{IAV-Induced Suppression of Macrophage Antibacterial} Function Is Alleviated in TLR7-Deficient Hosts

IAV infection has previously been shown to desensitize AM to bacterial TLR-ligands. This in turn led to an

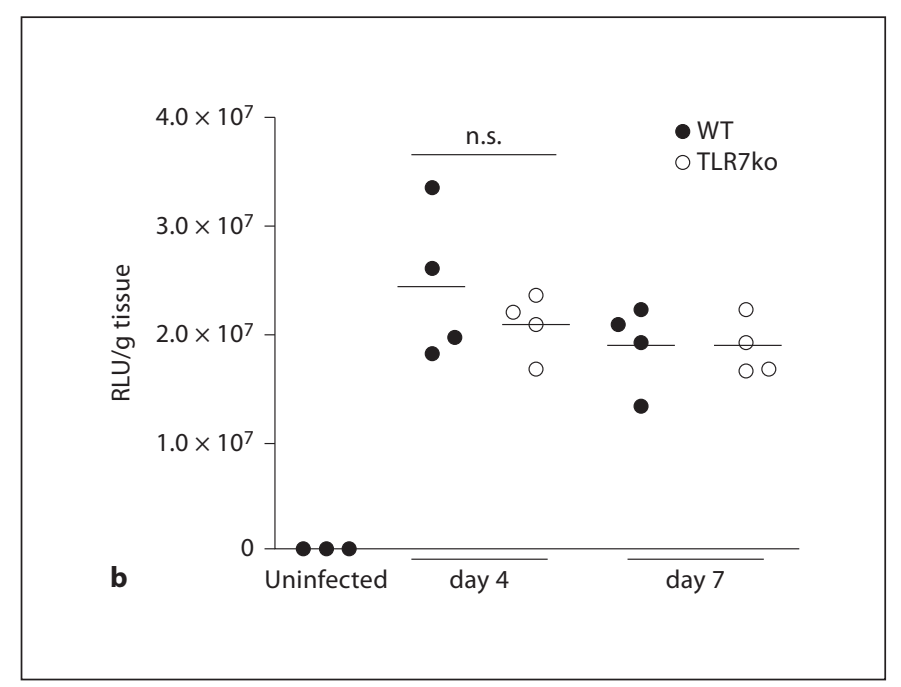

reporter mice were sacrificed at the indicated time points following IAV infection. Luciferase activity in lungs was quantified ex vivo and is shown as relative light units (RLU)/g tissue for individual mice from one representative out of two experiments. Data were compared by an unpaired, two-sided t test.

inhibition of neutrophil recruitment in response to a secondary bacterial challenge [5]. In order to assess whether this also held true for our co-infection model and whether TLR7 played a role in this mechanism, we characterized the recruitment of effector cells to the airways following pneumococcal infection in naïve mice and mice pre-infected with IAV. In contrast to Didierlaurent et al. [5] we found the number of cells present in BAL $20 \mathrm{~h}$ following pneumococcal infection on day 7 after influenza to be strongly and significantly increased compared to that after S. pneumoniae single infection (fig. 6a). The total cell numbers as well as the numbers of neutrophils and macrophages recruited to the airways did not differ between WT and TLR7ko mice, irrespective of the presence and timing of IAV pre-infection (fig. 6a). Thus, an altered recruitment of effector cells following co-infection could not underlie the differences in disease course we found in TLR7-deficient hosts.

IFN- $\gamma$ produced during the anti-IAV response decreases AM scavenger receptor expression and inhibits AM phagocytic activity in secondary bacterial infection [7]. To test whether such a phenomenon was responsible for the effects seen in TLR7ko mice, we assessed AM function in 


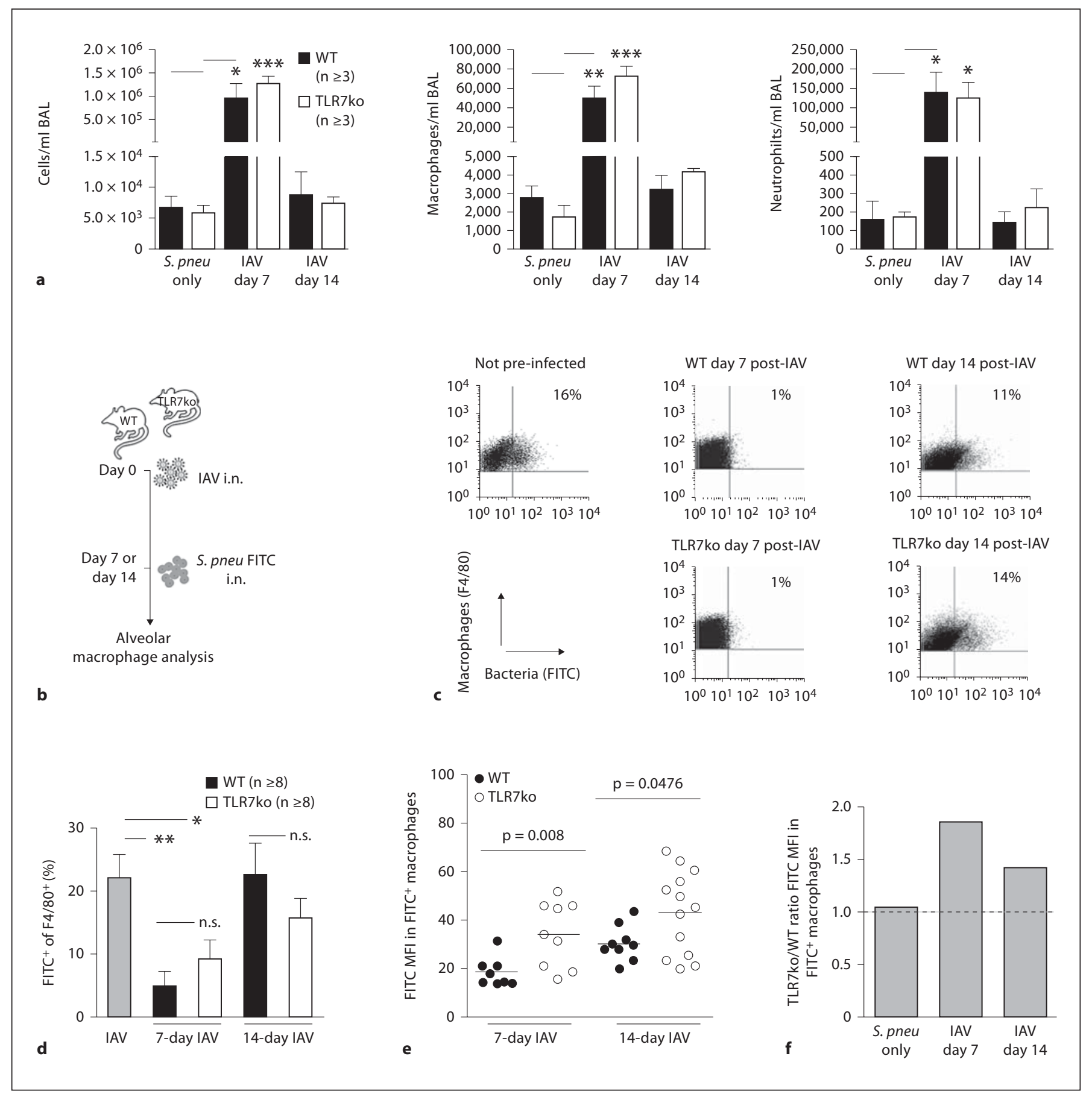

Fig. 6. Suppression of macrophage function during IAV infection is independent of TLR7 but alleviated in TLR7ko hosts. a WT (solid bars) and TLR7ko (open bars) mice were infected with $1 \times$ $10^{5}$ CFU S. pneumoniae without previous IAV infection or on day 7 or 14 after infection with 0.04 LD $_{50}$ IAV PR8 and were sacrificed $20 \mathrm{~h}$ later. The total number of cells present in bronchoalveolar lavage was determined and the numbers of macrophages and neutrophils were quantified by flow-cytometry. Data show results of one representative out of two experiments with $n \geq 3$ mice/group. b An in vivo phagocytosis assay was performed to assess phago- cytic activity of AM without preceding IAV infection and 7 or 14 days following PR8 infection. Flow-cytometric analysis (c) was performed to quantify the proportion of $\mathrm{FITC}^{+} / \mathrm{F} 4 / 80^{+}$cells out of all $\mathrm{F} 4 / 80^{+}$macrophages (d) and the respective FITC mean fluorescence intensity (MFI) (e). Groups were compared by an unpaired, two-sided t test. $\mathbf{f}$ The ratio of TLR7ko over WT FITC MFI of FITC ${ }^{+}$AM was assessed by dividing the mean MFI value of the TLR7ko groups by the value of the corresponding WT group. n.s. $=$ Not significant. 
co-infected WT and TLR7ko mice. This was especially interesting as the early airway IFN- $\gamma$ response after IAV infection was inhibited in TLR7-deficient hosts (fig. 2a). We found that expression of the class A scavenger receptor MARCO was reduced on AM from 7-day IAV-infected WT and TLR7ko mice to the same extent (data not shown). Thus, we also performed in vivo phagocytosis assays in WT and TLR7-deficient mice through intranasal administration of fluorescently (FITC-)labeled S. pneumoniae (fig. 6b). Indeed, AM of 7 days IAV-pre-infected mice displayed a significantly reduced ability to phagocytose $S$. pneumoniae in the lung. This was mirrored by a reduced frequency of $\mathrm{FITC}^{+}$macrophages isolated from these animals (fig. 6c, d). This was the case for both WT and TLR7ko mice to the same extent and both mouse strains had partly recovered from this functional suppression by day 14 after the viral infection (fig. 6d). However, when we compared the FITC mean fluorescence intensity as a quantitative measure of the amount of bacteria that macrophages had bound or taken up, AM isolated from TLR7ko animals, irrespective of whether they had been IAV infected 1 or 2 weeks before, displayed significantly higher values after encounter of FITC-labeled S. pneumoniae than those from WT mice (day $7 \mathrm{p}=0.008$, day $14 \mathrm{p}=0.0476$; fig. 6e). In contrast, the mean fluorescence intensities that were obtained when the assay was performed in uninfected WT and TLR7ko mice were comparable between both mouse strains (fig. 6f). Taken together, these results demonstrated that the overall AM phagocytic capacity was inhibited 7 days following IAV infection irrespective of the presence of TLR7. However, AM from TLR7-deficient hosts were less strongly inhibited in binding and phagocytosis of $S$. pneumoniae both 7 and 14 days after IAV infection. Even though not sufficient to protect animals from enhanced susceptibility to bacterial superinfection during acute IAV, this possibly at least in part explains the delayed progression of secondary bacterial disease during acute influenza which we observed in TLR7ko hosts.

\section{Discussion}

In this study we have addressed the role of TLR7 in the immune response towards bacterial superinfection. In our mouse model of IAV infection alone, TLR7 deficiency neither had an impact on the outcome of the infection nor on clearance of the virus. This is in line with findings from a study by Koyama et al. [19], where signaling by the PRR RIG-I was found to compensate for TLR7 deficiency in the initial response to IAV infection. As recognition of one pattern by different receptors offers additional opportunities for orchestrating the triggered responses, it is highly conceivable that TLR7 plays a role in fine-tuning initial responses, as shown for IFN- $\gamma$ production, and adaptive anti-IAV immunity [16-19], rather than constituting a receptor which is responsible for the induction of an effective defense alone.

Next to the resolution of the viral infection as such, protection against secondary bacterial pathogens plays a crucial role in IAV infections. This is implied by the high incidence of severe disease and deaths through secondary infections in IAV-infected patients as observed for past and present pandemics. During recent years, various studies have addressed this synergism between viral and bacterial pathogens and have identified several ways by which viral infections modulate the defense against secondary bacterial infections [5-9, 34-38]. The detailed underlying mechanisms, however, still remain incompletely understood. Up to now, studies addressing the role of PRRs in conferring enhanced susceptibility to bacterial superinfection after influenza have selectively addressed the role of bacteria-sensing receptors. TLR2 has been shown not to play a role in the induction of this phenomenon directly [39], whereas others demonstrated that IAV infection leads to an impressively long-lasting general desensitization of the bacteria-recognizing TLR 2, 4 and 5. This in consequence facilitates secondary bacterial infections [5]. However, to our knowledge it has not been considered that also a virus-sensing PRR might affect host responses towards subsequent bacterial infections. A distinct role of TLR7 becomes especially conceivable when taking into account that most viruses predisposing for bacterial superinfection carry a single-stranded RNA genome, thus providing a common innate stimulus $[21,22]$. Supporting such a concept, we show here that a virally triggered PRR indeed affects the defense against a secondary bacterial pathogen. While TLR7 deficiency does not drastically impact the effectiveness of anti-IAV responses, it delayed the progression of a secondary pneumococcal infection. Ultimately however, this failed to protect from high mortality to lowdose S. pneumoniae infection following IAV, thereby highlighting that the influence of TLR7-dependent factors in this synergism is only little and most likely inferior to other mechanisms playing a role.

Type I IFNs can modulate host immunity in several ways $[6,40]$ and distinct aspects of this modulation are achieved also through selective triggering of TLR7 by synthetic ligands $[14,26]$. During in vivo IAV infection, the induction and kinetics of the overall type I IFN response are not exclusively mediated by TLR7, but also through 
triggering of additional innate immune receptors (fig. 5) $[19,33]$. Therefore, the presence of high levels of type I IFNs following IAV infection also in TLR7-deficient hosts could at least in part explain why the demonstrated contribution of TLR7 triggering to enhanced susceptibility to bacterial superinfection is not able to protect from the fatal outcome.

Another mechanism by which influenza virus infection has been shown to mediate enhanced susceptibility to bacterial superinfection is by modulating the effectivity of antibacterial functions of AM. A reduced capacity of these cells to produce chemokines and recruit neutrophils in response to bacterial TLR-triggering and compromised antibacterial phagocytic functions have both been shown to be consequences of influenza virus infection [5, 7]. IFN- $\gamma$ produced during the viral infection was found to mediate the suppression of AM phagocytosis during secondary bacterial infection, which in turn led to uncontrolled outgrowth of the bacteria in pre-infected animals [7]. Our findings suggest that TLR7 deficiency has an impact on this IAV-induced suppression of macrophage function as we found AM from IAV-infected TLR7ko mice to bind and take up S. pneumoniae more efficiently both during acute viral infection and after its resolution. AM have been found to be crucial for ensuring sterility of the lower respiratory tract and they are key players in the early defense against $S$. pneumoniae infection [41]. They fulfill this role by the binding, uptake and killing of bacteria. The disadvantage we found for AM of IAV-pre-infected WT compared to TLR7ko mice in binding and phagocytosing pneumococci in the respiratory tract might directly or indirectly have led to the higher bacterial numbers found in the lungs of WT mice $24 \mathrm{~h}$ following co-infection on day 7 after IAV infection. In a second step of the anti-bacterial defense neutrophils are recruited to the respiratory tract, a process we found to be unaltered when comparing co-infected mice of both strains. However, the strong bacterial outgrowth in the lungs of coinfected animals apparently overwhelmed the recruited cells which were not able to prevent further spread of the bacteria in either WT or TLR7-deficient hosts. It is possible that, even though the lung bacterial burden did not differ significantly between the two mouse strains, the critical threshold of lung bacterial outgrowth, at which bacteria start to spread from the respiratory tract throughout the organism via the bloodstream, was reached at a significantly earlier time-point in co-infected WT rather than TLR7ko mice. Therefore, in the absence of TLR7, a less severe inhibition of AM following IAV infection possibly accounts for the reduced bacterial burden found in the lungs of co-infected mice and the significantly delayed systemic dissemination of bacteria as well as the absence of long-term immune suppression.

Even though the effects of the virus-sensing PRR TLR7 on post-influenza anti-bacterial defense are slight and a lack of TLR7 did not lead to enhanced survival in bacterial superinfection, our study adds a new aspect to the increasing understanding of the mechanisms underlying the synergism between IAV and S. pneumoniae. However, further studies will be needed to identify the routes through which TLR7 triggering by IAV influences the rapid progression of secondary bacterial disease. Clearly, a detailed knowledge of all of the involved mechanisms as well as the relative importance of their impact will be necessary to face this dangerous threat to human health and to be able to exploit all possible aspects for future strategies of prevention and treatment, possibly including the option of modulating PRR-signaling. This is well demonstrated by studies regarding TLR2, which, even though previously shown not to influence susceptibility to severe secondary bacterial disease after influenza [39], was later found to actually play an important role in mediating immunopathology during its treatment [42].

\section{Acknowledgements}

We thank H. Frauendorf (Immuneregulation, Helmholtz Centre for Infection Research, Braunschweig) for the preparation of the pVI-PmH5-PR8-NP plasmid standard.

This work was supported by the International Research Training Group 1273 and by the grant BR2221/1-1/GU769/5-1, both funded by the German Research Foundation (DFG). D.B. is supported by the President's Initiative and Networking Fund of the Helmholtz Association of German Research Centers (HGF) under contract No. W2/W3-029.

References

$\checkmark 1$ Morens DM, Taubenberger JK, Fauci AS: Predominant role of bacterial pneumonia as a cause of death in pandemic influenza: implications for pandemic influenza preparedness. J Infect Dis 2008;198:962-970.

2 Centers for Disease Control and Prevention: Bacterial coinfections in lung tissue specimens from fatal cases of 2009 pandemic influenza A (H1N1) - United States, May-August 2009. MMWR Morb Mortal Wkly Rep 2009;58:1071-1074.

-3 Estenssoro E, Rios FG, Apezteguia C, Reina R, Neira J, Ceraso DH, Orlandi C, Valentini R, Tiribelli N, Brizuela M, Balasini C, Mare S, Domeniconi G, Ilutovich S, Gomez A, Giuliani J, Barrios C, Valdez P: Pandemic 2009 influenza A in Argentina: a study of 337 patients on mechanical ventilation. Am J Respir Crit Care Med 2010;182:41-48. 
-4 Palacios G, Hornig M, Cisterna D, Savji N, 17 Heer AK, Shamshiev A, Donda A, Uematsu Bussetti AV, Kapoor V, Hui J, Tokarz R, Briese T, Baumeister E, Lipkin WI: Streptococcus pneumoniae coinfection is correlated with the severity of H1N1 pandemic influenza. PLoS One 2009;4:e8540.

-5 Didierlaurent A, Goulding J, Patel S, Snelgrove R, Low L, Bebien M, Lawrence T, van Rijt LS, Lambrecht BN, Sirard JC, Hussell T: Sustained desensitization to bacterial tolllike receptor ligands after resolution of respiratory influenza infection. J Exp Med 2008; 205:323-329.

-6 Shahangian A, Chow EK, Tian X, Kang JR, Ghaffari A, Liu SY, Belperio JA, Cheng G, Deng JC: Type I IFNs mediate development of postinfluenza bacterial pneumonia in mice. J Clin Invest 2009;119:1910-1920.

7 Sun K, Metzger DW: Inhibition of pulmonary antibacterial defense by interferongamma during recovery from influenza infection. Nat Med 2008;14:558-564.

8 van der Sluijs KF, van Elden LJ, Nijhuis M, Schuurman R, Pater JM, Florquin S, Goldman M, Jansen HM, Lutter R, van der Poll T: IL-10 is an important mediator of the enhanced susceptibility to pneumococcal pneumonia after influenza infection. J Immunol 2004;172:7603-7609.

9 Zavitz CC, Bauer CM, Gaschler GJ, Fraser KM, Strieter RM, Hogaboam CM, Stampfli MR: Dysregulated macrophage-inflammatory protein-2 expression drives illness in bacterial superinfection of influenza. J Immunol 2010;184:2001-2013.

10 Akira S, Takeda K: Functions of toll-like receptors: lessons from $\mathrm{KO}$ mice. C R Biol 2004;327:581-589.

11 Kawai T, Akira S: The role of pattern-recognition receptors in innate immunity: update on toll-like receptors. Nat Immunol 2010;11: 373-384.

-12 Diebold SS, Kaisho T, Hemmi H, Akira S, Reis e Sousa: Innate antiviral responses by means of TLR7-mediated recognition of singlestranded RNA. Science 2004;303:1529-1531.

-13 Heil F, Hemmi H, Hochrein H, Ampenberger F, Kirschning C, Akira S, Lipford G, Wagner H, Bauer S: Species-specific recognition of single-stranded RNA via toll-like receptor 7 and 8. Science 2004;303:1526-1529.

- 14 Gunzer M, Riemann H, Basoglu Y, Hillmer A, Weishaupt C, Balkow S, Benninghoff B, Ernst B, Steinert M, Scholzen T, Sunderkotter C, Grabbe S: Systemic administration of a TLR7 ligand leads to transient immune incompetence due to peripheral-blood leukocyte depletion. Blood 2005;106:2424-2432.

- 15 Xirakia C, Koltsida O, Stavropoulos A, Thanassopoulou A, Aidinis V, Sideras P, Andreakos E: Toll-like receptor 7-triggered immune response in the lung mediates acute and long-lasting suppression of experimental asthma. Am J Respir Crit Care Med 2010 181:1207-1216.

16 Geeraedts F, Goutagny N, Hornung V, Severa M, de HA, Pool J, Wilschut J, Fitzgerald KA, Huckriede A: Superior immunogenicity of inactivated whole virus $\mathrm{H} 5 \mathrm{~N} 1$ influenza vaccine is primarily controlled by toll-like receptor signalling. PLoS Pathog 2008;4:e1000138.
S, Akira S, Kopf M, Marsland BJ: TLR signaling fine-tunes anti-influenza $B$ cell responses without regulating effector $\mathrm{T}$ cell responses. J Immunol 2007;178:2182-2191.

18 Jeisy-Scott V, Davis WG, Patel JR, Bowzard JB, Shieh WJ, Zaki SR, Katz JM, Sambhara S: Increased MDSC accumulation and Th2 biased response to influenza $\mathrm{A}$ virus infection in the absence of TLR7 in mice. PLoS One 2011;6:e25242.

19 Koyama S, Ishii KJ, Kumar H, Tanimoto T, Coban C, Uematsu S, Kawai T, Akira S: Differential role of TLR- and RLR-signaling in the immune responses to influenza A virus infection and vaccination. J Immunol 2007; 179:4711-4720

20 Seo SU, Kwon HJ, Song JH, Byun YH, Seong BL, Kawai T, Akira S, Kweon MN: MyD88 signaling is indispensable for primary influenza A virus infection but dispensable for secondary infection. J Virol 2010;84:12713-12722.

21 McCullers JA: Insights into the interaction between influenza virus and pneumococcus. Clin Microbiol Rev 2006;19:571-582.

22 Techasaensiri B, Techasaensiri C, Mejias A, McCracken GH Jr., Ramilo O: Viral coinfections in children with invasive pneumococcal disease. Pediatr Infect Dis J 2010;29:519523.

23 Lukacs NW, Smit JJ, Mukherjee S, Morris SB, Nunez G, Lindell DM: Respiratory virus-induced TLR7 activation controls IL-17-associated increased mucus via IL-23 regulation. J Immunol 2010;185:2231-2239.

24 Triantafilou K, Vakakis E, Richer EA, Evans GL, Villiers JP, Triantafilou M: Human rhinovirus recognition in non-immune cells is mediated by toll-like receptors and MDA-5, which trigger a synergetic pro-inflammatory immune response. Virulence 2011;2:22-29.

$\checkmark 25$ Hemmi H, Kaisho T, Takeuchi O, Sato S, Sanjo H, Hoshino K, Horiuchi T, Tomizawa H, Takeda K, Akira S: Small anti-viral compounds activate immune cells via the TLR7 MyD88-dependent signaling pathway. Nat Immunol 2002;3:196-200.

26 Stegemann S, Dahlberg S, Kroger A, Gereke M, Bruder D, Henriques-Normark B, Gunzer M: Increased susceptibility for superinfection with Streptococcus pneumoniae during influenza virus infection is not caused by TLR7-mediated lymphopenia. PLoS One2009; 4:e4840.

27 Lienenklaus S, Cornitescu M, Zietara N, Lyszkiewicz M, Gekara N, Jablonska J, Edenhofer F, Rajewsky K, Bruder D, Hafner M, Staeheli P, Weiss S: Novel reporter mouse reveals constitutive and inflammatory expression of IFN- $\beta$ in vivo. J Immunol 2009;183: 3229-3236.

28 Dessing MC, van der Sluijs KF, Spek CA, van der Poll T: Gene expression profiles in murine influenza pneumonia. J Innate Immun 2009;1:366-375.

29 Julkunen I, Sareneva T, Pirhonen J, Ronni T, Melen K, Matikainen S: Molecular pathogenesis of influenza A virus infection and virus-induced regulation of cytokine gene expression. Cytokine Growth Factor Rev 2001;12:171-180.
30 Moltedo B, Lopez CB, Pazos M, Becker MI, Hermesh T, Moran TM: Cutting edge: stealth influenza virus replication precedes the initiation of adaptive immunity. J Immunol 2009; 183:3569-3573.

31 Rothberg MB, Haessler SD: Complications of seasonal and pandemic influenza. Crit Care Med 2010;38:e91-e97.

32 Sandgren A, Albiger B, Orihuela CJ, Tuomanen E, Normark S, Henriques-Normark $B$ : Virulence in mice of pneumococcal clonal types with known invasive disease potential in humans. J Infect Dis 2005;192:791800.

33 Ehrhardt C, Seyer R, Hrincius ER, Eierhoff $\mathrm{T}$, Wolff $\mathrm{T}$, Ludwig S: Interplay between influenza A virus and the innate immune signaling. Microbes Infect 2010;12:81-87.

- 34 Jamieson AM, Yu S, Annicelli CH, Medzhitov R: Influenza virus-induced glucocorticoids compromise innate host defense against a secondary bacterial infection. Cell Host Microbe 2010;7:103-114.

35 Kudva A, Scheller EV, Robinson KM, Crowe CR, Choi SM, Slight SR, Khader SA, Dubin PJ, Enelow RI, Kolls JK, Alcorn JF: Influenza A inhibits Th17-mediated host defense against bacterial pneumonia in mice. J Immunol 2011;186:1666-1674.

36 McNamee LA, Harmsen AG: Both influenza-induced neutrophil dysfunction and neutrophil-independent mechanisms contribute to increased susceptibility to a secondary Streptococcus pneumoniae infection. Infect Immun 2006;74:6707-6721.

37 Navarini AA, Recher M, Lang KS, Georgiev P, Meury S, Bergthaler A, Flatz L, Bille J, Landmann R, Odermatt $B$, Hengartner $H$, Zinkernagel RM: Increased susceptibility to bacterial superinfection as a consequence of innate antiviral responses. Proc Natl Acad Sci USA 2006;103:15535-15539.

- 38 Small CL, Shaler CR, McCormick S, Jeyanathan M, Damjanovic D, Brown EG, Arck P, Jordana M, Kaushic C, Ashkar AA, Xing Z: Influenza infection leads to increased susceptibility to subsequent bacterial superinfection by impairing $\mathrm{NK}$ cell responses in the lung. J Immunol 2010;184:2048-2056.

- 39 Dessing MC, van der Sluijs KF, Florquin S, Akira S, van der Poll T: Toll-like receptor 2 does not contribute to host response during postinfluenza pneumococcal pneumonia. Am J Respir Cell Mol Biol 2007;36:609-614.

- 40 Kamphuis E, Junt T, Waibler Z, Forster R, Kalinke U: Type I interferons directly regulate lymphocyte recirculation and cause transient blood lymphopenia. Blood 2006; 108:3253-3261.

-41 Sun K, Gan Y, Metzger DW: Analysis of murine genetic predisposition to pneumococcal infection reveals a critical role of alveolar macrophages in maintaining the sterility of the lower respiratory tract. Infect Immun 2011;79:1842-1847.

42 Karlstrom A, Heston SM, Boyd KL, Tuomanen EI, McCullers JA: Toll-like receptor 2 mediates fatal immunopathology in mice during treatment of secondary pneumococcal pneumonia following influenza. J Infect Dis 2011;204:1358-1366. 